\title{
SARS-CoV-2 Variants of Interest and Concern naming scheme conducive for global discourse
}

\author{
A group convened and led by the Virus Evolution Working Group of the World Health Organization reports on its \\ deliberations and announces a naming scheme that will enable clear communication about SARS-CoV-2 variants of \\ interest and concern.
}

\author{
Frank Konings, Mark D. Perkins, Jens H. Kuhn, Mark J. Pallen, Erik J. Alm, Brett N. Archer, Amal Barakat, \\ Trevor Bedford, Jinal N. Bhiman, Leon Caly, Lisa L. Carter, Anne Cullinane, Tulio de Oliveira, \\ Julian Druce, Ihab El Masry, Roger Evans, George F. Gao, Alexander E. Gorbalenya, Esther Hamblion, \\ Belinda L. Herring, Emma Hodcroft, Edward C. Holmes, Manish Kakkar, Shagun Khare, \\ Marion P. G. Koopmans, Bette Korber, Juliana Leite, Duncan MacCannell, Marco Marklewitz, \\ Sebastian Maurer-Stroh, Jairo Andres Mendez Rico, Vincent J. Munster, Richard Neher, \\ Bas Oude Munnink, Boris I. Pavlin, Malik Peiris, Leo Poon, Oliver Pybus, Andrew Rambaut, \\ Paola Resende, Lorenzo Subissi, Volker Thiel, Suxiang Tong, Sylvie van der Werf, Anne von Gottberg, \\ John Ziebuhr and Maria D. Van Kerkhove
}

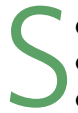
evere acute respiratory syndrome coronavirus 2 (SARS-CoV-2), the causative agent of coronavirus disease 2019 (COVID-19), has a linear, unsegmented, positive-sense RNA genome. As with all viruses, SARS-CoV-2 continuously adapts to changing environments in real time via random genome mutations that are subject to natural selection. Most mutations are neutral or detrimental to the virus; however, a small number of mutations may provide a selective advantage, such as escape from the host immune system or resistance to antiviral drugs. Such mutations may also lead to increased fitness for transmissibility. As mutated forms of viruses or variants spread from person to person, they will eventually be detected at the population level.

The World Health Organization (WHO) COVID-19 Reference Laboratory Network $^{1}$ has been tracking SARS-CoV-2 mutations since the beginning of the pandemic. In June 2020, the WHO Virus Evolution Working Group was established with a specific focus on SARS-CoV-2 variants, their phenotype and their impact on countermeasures. The WHO has developed a global risk-monitoring framework to coordinate components of an international system for monitoring and assessing SARS-CoV-2 variants and their impact. Specifically, this framework aims to collect, analyse and share data to identify crucial priorities, set triggers for decision making and enable and improve the capacities of laboratories, technical networks and expert groups.
The WHO has provided working definitions of variants of interest (VOIs) and variants of concern (VOCs) that will be updated when required ${ }^{2}$. These working definitions also consider changes that lead to an altered phenotype, which are harder to reflect in genotype-based classifications. Additionally, the WHO has advised how VOIs and VOCs should be reported to both national health authorities and to the organization itself. Initial cases or clusters associated with a VOC infection should be reported to the WHO through the International Health Regulations (2005) mechanism. The WHO has outlined actions required by its member states, reference laboratories and the $\mathrm{WHO}$ secretariat to assess the impact of VOIs and VOCs on the epidemiology and severity of COVID19 , and on the performance of available diagnostics, therapeutics, vaccines and public health initiatives. At the time of writing, several VOIs and VOCs are being tracked ${ }^{3}$.

\section{Current naming systems for SARS-CoV-2 variants}

Three nomenclature systems for naming and tracking SARS-CoV-2 genetic lineages are currently in use: the Global Initiative on Sharing All Influenza Data (GISAID) ${ }^{4}$, Nextstrain ${ }^{5}$ and Pango ${ }^{6}$. Each system has its own scientific approach to classify and name lineages, and all three systems had been introduced before VOIs and VOCs were recognized. The existence of different nomenclature systems can mean that the same variant has multiple names, often at the same time and without regard for the properties of VOIs and VOCs. The lack of clarity on naming VOCs makes it difficult for those who are not experts in the field to link such variants to scientific publications. Of similar concern, the use of multiple nomenclature systems is confusing for health officials, the media and the public, and undermines effective liaison between all stakeholders who must communicate easily to reach swift decisions on matters of public health concern.

It has become clear that the naming of SARS-CoV-2 variants is not trivial for several reasons. First, there is no centralized authority or process responsible for VOI and VOC naming, or a consensus on their designating criteria, meaning that different groups have assigned different names to the same variant. Second, a name that includes the location where a VOI or VOC was first detected can stigmatize places, countries and their populations, and could negatively impact surveillance and reporting of VOIs or VOCs. In addition, using the name of a place where a VOI or VOC was first detected is misleading - a VOI or VOC may have actually originated elsewhere, perhaps in a country with reduced sequencing capacity. Finally, alphanumeric naming schemes have resulted in complex names that are liable to misreporting and misunderstanding. Even a small mistake (such as typing ' 1 ' instead of '2' or placing dots incorrectly) in a numerical variant name or list of names can create confusion. 
Table 1 | The WHO naming mechanism for SARS-CoV-2 VOIs and VOCs

\begin{tabular}{|c|c|c|c|c|c|c|c|}
\hline $\begin{array}{l}\text { WHO } \\
\text { label }\end{array}$ & $\begin{array}{l}\text { Variant } \\
\text { type }\end{array}$ & $\begin{array}{l}\text { Country of first } \\
\text { detection }\end{array}$ & Date of designation & $\begin{array}{l}\text { Earliest documented } \\
\text { samples }\end{array}$ & $\begin{array}{l}\text { GISAID clade/ } \\
\text { variant }\end{array}$ & Nextstrain clade & $\begin{array}{l}\text { Pango } \\
\text { lineage }\end{array}$ \\
\hline Alpha & VOC & United Kingdom & 18 December 2020 & September 2020 & $\begin{array}{l}\text { GRY (formerly } \\
\text { GR/501Y.V1) }\end{array}$ & 20I/501Y.V1 & B.1.1.7 \\
\hline Beta & VOC & South Africa & 18 December 2020 & May 2020 & GH/501Y.V2 & 20H/501Y.V2 & B.1.351 \\
\hline Gamma & VOC & Brazil & 11 January 2021 & November 2020 & GR/501Y.V3 & 20J/501Y.V3 & P.1 \\
\hline Delta & VOC & India & $\begin{array}{l}4 \text { April } 2021 \text { (VOI); } 11 \\
\text { May } 2021 \text { (VOC) }\end{array}$ & October 2020 & G/452R.V3 & 21A/S:478K & B.1.617.2 \\
\hline
\end{tabular}

A continuously updated list of names can be found at https://www.who.int/activities/tracking-SARS-CoV-2-variants.

\section{Outputs of the discussion to improve the naming of VOIs and VOCs}

Only some of the variants that are named differently in the three existing systems are VOIs or VOCs, and new names need to be prioritized for these. To formulate an improved naming scheme for VOIs and VOCs, the WHO invited groups that have published phylogenetics-based classification and nomenclature systems for SARS-CoV-2 variants, and experts in virological and microbial nomenclature, to find possible solutions to these challenges. Participants drew the following conclusions:

(1) Existing naming systems are based on genetic information and do not always consider changes in the biological properties of variants. Currently, no VOI and VOC naming strategy has been standardized to enable straightforward communication. The existence of multiple technical VOI and VOC names results in confusion among political decision makers and the public.

(2) A mechanism using a prefix (for example, 'V' or 'VAR') combined with a consecutive number assigned to each new variant is unsuitable because it would result in similar names to those used by existing nomenclature systems; that is, a combination of letters and numbers. Using neutral names produced by an algorithm that takes existing words, extracts useful syllables and recombines them in a combinatorial fashion was also explored. However, using this method, it was not possible to easily generate sufficient numbers of two- or three-syllable words that were easy to pronounce, acceptable to the majority of experts consulted, not already named after a person, place or company, and not otherwise registered in the World Intellectual Property Organization Global Brand Database.

(3) Having a label that is less scientific would be useful. This includes avoiding a label that uses a combination of letters and numbers, such as those used by existing naming systems based on genomic changes. Mechanisms that use any kind of geographic reference - even those using an approach with coded locations that could potentially be deciphered - were discarded. All parties present agreed to create and use a new WHO labelling mechanism primarily for VOIs and VOCs.

\section{WHO naming mechanism for VOls and VOCs}

As soon as variants are designated as VOIs and VOCs according to the WHO working definitions, labels will be chosen and applied. When working definitions are revised - for example, when other characteristics of the VOI or VOC are included - the labelling mechanism will follow the new definitions. Labels will be selected by the WHO using names of Greek alphabet letters: alpha, beta, gamma, and so on. These will be easier to remember and more practical to use than alphanumerical designations. The Greek alphabet is well established as being generic, as the names of its individual letters have already been used for a multitude of purposes. While some of these names may also be the name of a person, place or company at present, there is no intent to establish a link with these and the mechanism is purely used to label the SARS-CoV-2 variants for ease of communication. Once all 24 letters have been assigned to VOIs or VOCs, other lists of names will be considered.

Labels for VOIs and VOCs, their links to existing phylogenetics-based SARS-CoV-2 classification and nomenclature systems, and their key scientific and medical features will be published and updated continuously by the WHO at https://www. who.int/activities/tracking-SARS-CoV2 -variants. It is recommended that all interested parties, especially journalists, visit this site frequently. Examples of VOI and VOC labels, along with their scientific designations in three different classification systems based on phylogenetics, are shown in Table 1.

Finally, SARS-CoV-2 variants that do not meet the WHO's definition of a VOI or VOC may not be labelled via this scheme, unless deemed necessary by the organization in consultation with the Virus Evolution Working Group, but can be tracked using the classification systems of GISAID, Nextstrain and Pango. The WHO labelling mechanism is independent of the existing SARS-CoV-2 lineage nomenclature systems but is informed by the available classifications and will be cross-linked with them. Countries will inform the WHO through established reporting channels, with supporting information about cases associated with VOIs or VOCs, using WHO working definitions. When a VOI or VOC is confirmed by the WHO, a label will be issued. This label will anchor the three different names arising from the three existing naming systems.

\section{Conclusion}

We emphasize that the existing phylogenetics-based nomenclature systems of SARS-CoV-2 lineages convey important information and continue to inform research. This new naming system is intended to facilitate the sharing of research advancements with a broader audience and aims to provide a platform to enable clear global discourse around VOIs and VOCs.

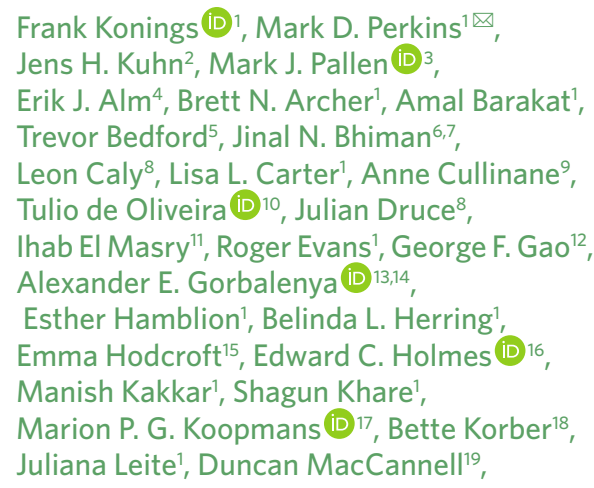


Marco Marklewitz',

Sebastian Maurer-Stroh ${ }^{20,21, J}$

airo Andres Mendez Rico',

Vincent J. Munster (D)22, Richard Neher (D) ${ }^{23}$,

Bas Oude Munnink (D)17, Boris I. Pavlin',

Malik Peiris (D24, Leo Poon (D24,

Oliver Pybus (D)25, Andrew Rambaut (D26,

Paola Resende (D) 27, Lorenzo Subissi (D),

Volker Thiel(D15,28, Suxiang Tong ${ }^{19}$,

Sylvie van der Werf (D) 29 ,

Anne von Gottberg ${ }^{6,7}$, John Ziebuhr (D) 30

and Maria D. Van Kerkhove ${ }^{1}$

${ }^{1}$ World Health Organization, Geneva, Switzerland. ${ }^{2}$ Integrated Research Facility at Fort Detrick, Division of Clinical Research, National Institute of Allergy and Infectious Diseases, National Institutes of Health, Fort Detrick, Frederick, MD, USA. ${ }^{3}$ Quadram Institute Bioscience, University of East Anglia, Norwich, UK. ${ }^{4}$ European Centre for Disease Prevention and Control, Stockholm, Sweden. ${ }^{5}$ Fred Hutchinson Cancer Research Center, Seattle, WA, USA. ${ }^{6}$ National Institute for Communicable Diseases, Johannesburg, South Africa. ${ }^{7}$ School of Pathology, Faculty of Health Sciences, University of the Witwatersrand, Johannesburg, South Africa. ${ }^{8}$ Victorian Infectious Diseases Reference Laboratory (VIDRL), Melbourne, Victoria, Australia. ${ }^{9}$ Faculty of Science and Engineering, University of Limerick, Limerick, Ireland. ${ }^{10}$ University of KwaZulu-Natal, Durban, South Africa. ${ }^{11}$ Emergency Centre for Transboundary Animal Disease, Food and Agriculture Organization of the United Nations (FAO), Cairo, Egypt. ${ }^{12}$ Chinese Center for Disease Control and Prevention, Beijing, P.R. China. ${ }^{13}$ Leiden University Medical Center, Leiden, The Netherlands. ${ }^{14}$ Faculty of Bioengineering and Bioinformatics, Lomonosov Moscow State University, Moscow, Russia. ${ }^{15}$ University of Bern, Bern, Switzerland. ${ }^{16}$ The University of Sydney, Sydney, New South Wales, Australia. ${ }^{17}$ Erasmus University, Rotterdam, The Netherlands. ${ }^{18}$ Theoretical Biology and Biophysics, Los Alamos National Laboratory, Los Alamos, NM, USA. ${ }^{19}$ United States Centers for Disease Control and Prevention, Atlanta, GA, USA. ${ }^{20}$ The Global Initiative on Sharing All Influenza Data (GISAID), Munich, Germany. ${ }^{21}$ Bioinformatics Institute, Agency for Science, Technology and Research, Singapore, Singapore. ${ }^{22}$ Virus Ecology Unit, National Institute of Allergy and Infectious Diseases, Rockville, MA, USA. ${ }^{23}$ Biozentrum, University of Basel, Switzerland Swiss Institute of Bioinformatics, Basel, Switzerland. ${ }^{24}$ The University of Hong Kong, Hong Kong, China. ${ }^{25}$ University of Oxford, Oxford, United Kingdom. ${ }^{26}$ Institute for Evolutionary Biology, Ashworth Laboratories, University of Edinburgh, Edinburgh, UK. ${ }^{27}$ Laboratory of Respiratory Viruses and Measles (LVRS), Oswaldo Cruz Institute, Fiocruz, Rio de Janeiro, Brazil. ${ }^{28}$ Institute of Virology and Immunology, Mittelhäusern, Switzerland. ${ }^{29}$ Institut Pasteur, Paris, France. ${ }^{30}$ Institute of Medical Virology,
Justus Liebig University Giessen, Giessen, Germany.

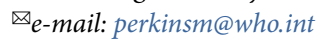

Published online: 9 June 2021

https://doi.org/10.1038/s41564-021-00932-w

References

1. WHO reference laboratories providing confirmatory testing for COVID-19. WHO https://go.nature.com/3ySxqX3 (2020).

2. COVID-19 weekly epidemiological update - 25 February 2021. WHO https://go.nature.com/3uEtXIj (2021).

3. Coronavirus disease (COVID-19) weekly epidemiological update and weekly operational update. WHO https://go.nature. com/3aahjbg (2021).

4. Clade and lineage nomenclature aids in genomic epidemiology studies of active $\mathrm{hCoV}-19$ viruses. GISAID https://go.nature. com/3pgSIt6 (2021).

5. Bedford, T., Hodcroft, E. B. \& Neher, R. A. Updated Nextstrain SARS-CoV-2 clade naming strategy. Nextstrain https://go.nature. com/3c9Riep (2021)

6. Rambaut, A. et al. Nat. Microbiol. 5, 1403-1407 (2020)

Acknowledgements

We thank D. Pereyaslov of the WHO Global Influenza Programme for their critical review of the manuscript. The views and conclusions contained in this document are those of the authors and should not be interpreted as necessarily representing the official policies, either expressed or implied, of the United States government, including those of the National Institutes of Health (NIH), the National Institute of Allergy and Infectious Diseases (NIAID), and the Centers for Disease Control and Prevention (CDC), or of the institutions and companies affiliated with the authors.

Competing interests

The authors declare no competing interests. 